\title{
POTENCIAIS BENEFÍCIOS DO AUMENTO DA TEMPERATURA DE ARMAZENAGEM EM ATMOSFERA CONTROLADA DE MAÇÃS 'GALA' TRATADAS COM 1-MCP'
}

\author{
ELIS REGINA MAZZURANA², LUIZ CARLOS ARGENTA ${ }^{3}$, \\ CASSANDRO VIDAL TALAMINI DO AMARANTE ${ }^{4}$, CRISTIANO ANDRÉ STEFFENS ${ }^{4}$
}

RESUMO - O armazenamento refrigerado pode induzir o desenvolvimento de danos por frio (caracterizado pelo escurecimento de polpa) em maçãs de algumas cultivares, mesmo quando mantidas em temperatura superior ao ponto de congelamento. $O$ presente estudo avaliou o aumento da temperatura de armazenagem de maçãs clones de 'Gala' sob atmosfera controlada (AC), tratadas com 1-metilciclopropeno (1-MCP), como método para redução do desenvolvimento de escurecimento da polpa e do consumo de energia durante o armazenamento. Experimentos foram conduzidos em 2011, com maçãs produzidas em Fraiburgo (armazenadas em câmaras experimentais a $-0,3$ e $1,2{ }^{\circ} \mathrm{C}$, e em câmaras comerciais a 0,7 e $2^{\circ} \mathrm{C}$ ) e em São Joaquim (armazenadas em câmaras comerciais a 0,$8 ; 1,4$ e $1,9^{\circ} \mathrm{C}$ ). Frutos de todos os tratamentos foram mantidos sob AC $\left(1,8 \pm 0,2 \mathrm{kPa}\right.$ de $\mathrm{O}_{2}$ e $2,0 \pm 0,2 \mathrm{kPa}$ de $\mathrm{CO}_{2} / \mathrm{UR}$ de $\left.91 \pm 4 \%\right)$, sendo que metade dos frutos de cada temperatura de armazenamento foi tratada com 1-MCP. Os frutos foram analisados após o armazenamento em AC quanto à firmeza da polpa e incidência de escurecimento da polpa, rachaduras e podridões. De maneira geral, maçãs armazenadas em temperaturas mais elevadas mantiveram melhor firmeza da polpa e apresentaram menor incidência de escurecimento da polpa rachaduras e podridões, quando não tratadas com 1-MCP. O tratamento com 1-MCP retardou a perda de firmeza de polpa e reduziu a ocorrência de escurecimento de polpa e rachaduras. $\mathrm{O}$ aumento da temperatura de armazenagem em câmaras comerciais de $\mathrm{AC}$ em Fraiburgo, de $0,7^{\circ} \mathrm{C}$ para $2,0^{\circ} \mathrm{C}$, resultou em economia no consumo de energia em aproximadamente $21 \%$ para ventilação do ar da câmara e $50 \%$ para refrigeração.

Termos para indexação: Malus domestica Borkh, temperatura, etileno, dano por frio, distúrbio fisiológico.

\section{POTENTIAL BENEFITS OF TEMPERATURE INCREASE DURING STORAGE UNDER CONTROLLED ATMOSPHERE OF 'GALA' APPLES TREATED WITH 1-MCP}

\begin{abstract}
Cold storage can induce the development of chilling injury (characterized by internal browning) in some apple cultivars, even when fruit are kept in temperature above the freezing point. This research evaluated the increment of storage temperature for 'Gala' apples under controlled atmosphere (CA) and 1-methylcyclopropene (1-MCP) treatment, as a method to reduce the development of internal browning and energy consumption. Experiments were conducted in 2011, with apples produced in the regions of Fraiburgo (stored in experimental chambers at -0.3 and $1.2{ }^{\circ} \mathrm{C}$, and in commercial chambers at 0.7 and 2 ${ }^{\circ} \mathrm{C}$ ) and São Joaquim (stored in commercial chambers at $0.8,1.4$ and $1.9^{\circ} \mathrm{C}$ ). Fruits of all treatments were kept under $\mathrm{CA}\left(1.8 \pm 0.2 \mathrm{kPa} \mathrm{O}_{2}\right.$ and $2.0 \pm 0.2 \mathrm{kPa} \mathrm{CO}_{2} / \mathrm{RH}$ of $\left.91 \pm 4 \%\right)$, with half of the fruits of each storage temperature being treated with 1-MCP. The fruit were assessed in terms of flesh firmness, and incidence of internal browning, skin cracking and decay. In general, apples stored at higher temperatures had better preservation of flesh firmness, and lower incidence of internal browning, skin cracking and decay, in fruit not treated with 1-MCP. The treatment with 1-MCP delayed flesh firmness loss and reduced the occurrence of internal browning and skin cracking. In Fraiburgo, increasing the AC storage temperature from $0.7^{\circ} \mathrm{C}$ to $2.0{ }^{\circ} \mathrm{C}$ resulted in energy saving of $\sim 21 \%$ for air ventilation and of $\sim 50 \%$ for cooling.

Index terms: Malus domestica Borkh, temperature, ethylene, chilling injury, physiological disorder.

${ }^{1}$ (Trabalho 237-14). Recebido para publicação em: 02-09-2015. Aceito para publicação em:09-11-2015.

${ }^{2}$ Mestranda em Produção Vegetal, Centro de Ciências Agroveterinárias (CAV), Universidade do Estado de Santa Catarina (UDESC), Av. Luiz de Camões, 2090, CEP 88520-000, Lages-SC. E-mail: elismazzurana@gmail.com

${ }^{3}$ Eng. Agr., D.Sc., Pesquisador da Empresa de Pesquisa Agropecuária e Extensão Rural e Santa Catarina S.A. (Epagri), Estação Experimental de Caçador, Cx. Postal 591, CEP 89500-000, Caçador, SC. E-mail: argenta@epagri.sc.gov.br

${ }^{4}$ Eng. Agr., Dr., Bolsista de Produtividade em Pesquisa do CNPq, Professor do Departamento de Agronomia, CAV/UDESC, Av. Luiz de Camões, 2090, CEP 88520-000, Lages-SC. E-mails: cassandro.amarante@udesc.br; cristiano.steffens@udesc.br
\end{abstract}




\section{INTRODUÇÃO}

Amaior parte da produção de maçãs é destinada à armazenagem, permitindo sua comercialização durante longos períodos após a colheita. A redução da temperatura é a ferramenta mais efetiva para estender a vida pós-colheita dos frutos, pois reduz a velocidade das reações bioquímicas associadas a maturação e senescência no fruto, incluindo a respiração e a produção de etileno (ERRAMPALLI et al., 2012). Adicionalmente, a refrigeração reduz o desenvolvimento de fungos patogênicos e a consequente deterioração por podridões após a colheita (KWEON et al., 2013). Entretanto, algumas cultivares de maçã podem desenvolver danos por frio ("chilling injury"), mesmo quando armazenadas em temperaturas acima de $0{ }^{\circ} \mathrm{C}$ (WANG, 2004). Temperaturas de armazenagem entre 0 e $1{ }^{\circ} \mathrm{C}$ tem sido recomentadas como ideais para a maioria das cultivares de maçãs, incluindo 'Gala', 'Golden Delicious', 'Red Delicious' e 'Fuji' (WATKINS et al., 2004).

O dano por frio ocorre após um período prolongado de exposição a baixas temperaturas, devido a perda da integridade de membranas, levando a descompartimentalização e extravasamento de solutos de organelas celulares (WANG, 2004). Em maçãs, normalmente o dano por frio se manifesta através do escurecimento da polpa, sendo a área afetada bem definida, geralmente farinácea e mais úmida que seca, e nos estágios iniciais o tecido com dano é separado da epiderme por uma área de tecido saudável (MEHERIUK et al., 1994).

Os impactos da temperatura de armazenagem sobre a conservação pós-colheita de várias cultivares de maçãs têm sido estudados há décadas, e embora a refrigeração de maçãs tenha papel fundamental na conservação da qualidade e prevenção de perdas da produção por deterioração, ela contribui significativamente para os custos da produção (BROWN, 2011; EAST et al., 2013). Adicionalmente, a refrigeração resulta em aumento da emissão de carbono, quando não se aplica fontes renováveis de energia (LIU et al., 2010).

A armazenagem de maçãs sob atmosfera controlada (AC), com baixa pressão parcial de oxigênio $\left(\mathrm{O}_{2}\right)$ e elevada pressão parcial de dióxido de carbono $\left(\mathrm{CO}_{2}\right)$, reduz a respiração e a produção e a ação do etileno, e retarda o amadurecimento, senescência e o desenvolvimento de vários distúrbios, especialmente aqueles relacionados a senescência, danos por frio (como é o caso da escaldadura na epiderme) e o desenvolvimento de fungos patogênicos (BRACKMANN et al., 2012). Além da
$\mathrm{AC}$, o tratamento de maçãs com 1-metilciclopropeno (1-MCP) vem sendo cada vez mais usado comercialmente, por efetivamente inibir a ação do etileno e controlar o amadurecimento e senescência de frutos (WATKINS, 2008; McCORMICK et al., 2010). Isto contribui na preservação da qualidade sensorial (McCORMICK et al., 2010), bem como reduz os distúrbios fisiológicos que se desenvolvem durante o armazenamento (WATKINS, 2007; WATKINS; MILLER, 2005). Por isso, a associação das tecnologias de refrigeração, AC e 1-MCP tem sido aplicada comercialmente, permitindo significativo aumento do potencial de conservação da qualidade dos frutos após a colheita (PRANGE et al., 2005).

$\mathrm{O}$ aumento da temperatura de armazenagem de maçãs armazenadas sob AC e tratadas com 1-MCP, pode proporcionar redução no consumo de energia, bem como na incidência de escurecimento interno da polpa (dano por frio), além de preservar os demais atributos de qualidade dos frutos (EAST et al., 2013). De acordo com Kweon et al. (2013), o aumento da temperatura de armazenagem de 0 ${ }^{\circ} \mathrm{C}$ para $2{ }^{\circ} \mathrm{C}$ em maçãs 'Fuji' armazenadas por seis meses reduziu a incidência de escurecimento interno da polpa. McCormick et al. (2012) também sugerem que é possível aumentar em $2,5^{\circ} \mathrm{C}\left(\mathrm{de} 1,5^{\circ} \mathrm{C}\right.$ para $4,0^{\circ} \mathrm{C}$ ) a temperatura de armazenagem em $\mathrm{AC}$, sem perda da qualidade de maçãs 'Gala', quando os frutos são tratados com 1-MCP. Brown (2011) reportou que o incremento na temperatura de armazenamento de 0 ${ }^{\circ} \mathrm{C}$ para $4,5^{\circ} \mathrm{C}$ em $\mathrm{AC}$ de 'Pink Lady', durante seis meses, não tem influência significativa na perda de firmeza da polpa dos frutos.

Este trabalho teve como objetivo avaliar a viabilidade do aumento na temperatura de armazenagem de maçãs clones de 'Gala' sob atmosfera controlada (AC), tratadas com 1-MCP, como método para redução do desenvolvimento de escurecimento da polpa e do consumo de energia durante o armazenamento.

\section{MATERIAL E MÉTODOS}

\section{Local de execução e condições de armazenamento dos frutos}

Os experimentos foram conduzidos com clones de maçãs 'Gala', produzidos na safra 20102011, em Fraiburgo, SC (27 $01^{\prime}$ S e $50^{\circ} 55^{\prime}$ W, com altitude de $1048 \mathrm{~m})$ e em São Joaquim, SC $\left(28^{\circ} 15^{\prime}\right.$ $\mathrm{S}$ e $49^{\circ} 54^{\prime} \mathrm{W}$, com altitude entre $1250 \mathrm{~m}$ ). Em Fraiburgo, um experimento foi realizado em câmaras de armazenagem experimental de $0,150 \mathrm{~m}^{3}$, e dois em câmaras comerciais de $2050 \mathrm{~m}^{3}$. Em São Joaquim, o 
experimento foi realizado em câmaras comerciais de $578 \mathrm{~m}^{3}$. Em todos os experimentos, os frutos foram armazenados em condição de $\mathrm{AC}\left(1,8 \pm 0,2 \mathrm{kPa}\right.$ de $\mathrm{O}_{2}$ e 2,0 $00,2 \mathrm{kPa}$ de $\mathrm{CO}_{2} /$ UR de $91 \pm 4 \%$ ).

Experimento 1: maçãs 'Royal Gala' colhidas em Fraiburgo e armazenadas em câmaras experimentais

$\mathrm{O}$ experimento foi conduzido com maçãs 'Royal Gala' crescidas sobre porta-enxerto EM7, em pomar comercial implantado em 1998, em Fraiburgo. As maçãs foram colhidas 124 e 140 dias após a plena floração (DAPF), e armazenadas sob AC, nas temperaturas de $-0,3 \pm 0,2{ }^{\circ} \mathrm{C}$ e $1,2 \pm 0,3{ }^{\circ} \mathrm{C}$, por 210 dias, em câmaras experimentais de $0,150 \mathrm{~m}^{3}$. Metade dos frutos de cada data de colheita foi tratada com 1-MCP $\left(1 \mu \mathrm{L} \mathrm{L}^{-1}\right)$, um dia após a colheita, e a outra metade permaneceu não tratada. As maçãs foram refrigeradas dois dias após a colheita, e expostas aos regimes de AC cinco dias após a colheita. Após o armazenamento em $\mathrm{AC}$, os frutos foram mantidos sob atmosfera do ar (AA; $23 \pm 0,3{ }^{\circ} \mathrm{C} / \mathrm{UR}$ de $68 \pm 6 \%$ ), por sete dias nos frutos colhidos aos $124 \mathrm{DAPF}$, e três dias nos frutos colhidos aos 140 DAPF.

$\mathrm{O}$ tratamento com 1-MCP foi feito em câmara hermética de $1 \mathrm{~m}^{3}$, a temperatura ambiente $\left(18-25^{\circ} \mathrm{C}\right)$, durante $24 \mathrm{~h}$. O gás 1-MCP foi gerado misturando-se pó de 1-metilciclopropeno-ciclodextrina (1,6 g de EthylBloc ${ }^{\mathrm{TM}}, 0,14 \%$ i.a., AgroFresh Inc.) e água a 25 ${ }^{\circ} \mathrm{C}$, num frasco de $500 \mathrm{~mL}$, e bombeado à câmara de tratamento em sistema fechado.

$\mathrm{Na}$ colheita, os frutos foram analisados quanto a firmeza da polpa e índice de iodo-amido. Após armazenamento em AC, seguido de AA a $23 \pm 0,3{ }^{\circ} \mathrm{C}$, os frutos foram analisados quanto a firmeza da polpa e severidade de escurecimento da polpa, podridões e rachaduras.

A firmeza da polpa foi medida em um lado da superfície de cada fruto, na região de transição de cor, do mais (mais exposto ao sol) ao menos avermelhado (menos exposto ao sol), pela utilização de um penetrômetro eletrônico, motorizado com ponteira de $11,1 \mathrm{~mm}$ (Güss, África do Sul), após remoção de uma pequena porção da epiderme. $O$ índice de iodo-amido foi avaliado numa escala de 1 (secção transversal da polpa corada com iodo, indicando alto teor de amido e fruto imaturo) a 9 (secção transversal da polpa não corada com iodo, indicando baixo teor de amido e fruto maduro).

Para avaliar a incidência de escurecimento interno (\%), os frutos foram cortados transversalmente na região equatorial e realizada a contagem dos que apresentavam a polpa com cor amarronzada/ pardacenta.

A incidência de podridões (\%) foi avaliada visualmente, sendo considerado fruto podre aquele que apresentava lesões com sintoma característico de ataque de patógenos.

A incidência de rachadura (\%) dos frutos foi analisada visualmente, considerando fruto rachado aquele que apresentavam rachaduras na epiderme e polpa.

Firmeza da polpa, índice de amido, severidade de podridões, escurecimento interno e rachaduras foram analisados para cada fruto.

$\mathrm{O}$ experimento foi conduzido segundo delineamento inteiramente casualizado, com quatro repetições de 50 frutos, em esquema fatorial $(2 \times 2)$, correspondente a duas temperaturas de armazenagem $\left(-0,3\right.$ e $\left.1,2^{\circ} \mathrm{C}\right)$ e duas doses de 1 -MCP $\left(0\right.$ e $\left.1 \mu \mathrm{L} \mathrm{L}^{-1}\right)$, para cada data de colheita.

Experimento 2: maçãs 'Galaxy' colhidas em Fraiburgo e armazenadas em câmaras comerciais.

$\mathrm{O}$ experimento foi conduzido com maçãs 'Galaxy' colhidas em pomares comerciais, em Fraiburgo, SC. Os pomares foram plantados em 2004, sobre porta-enxerto Marubakaido, com filtro EM9.

Este experimento foi conduzido em duas câmaras comercias de $2050 \mathrm{~m}^{3}$, ajustadas nas temperaturas de $0,7 \pm 0,3{ }^{\circ} \mathrm{C}$ e $2,0 \pm 0,3{ }^{\circ} \mathrm{C}$, posicionadas no meio de um mesmo corredor de 20 câmaras do mesmo tamanho. Nenhuma das duas câmaras apresentava parede exposta ao sol. Ambas as câmaras foram carregadas com $\sim 40$ toneladas de maçãs 'Galaxy', dos mesmos pomares de Fraiburgo, colhidas nos mesmos dias, durante cinco dias (134 a 140 DAPF). As cargas de maçãs de cada caminhão, destinadas a essas duas câmaras, foram divididas, sendo metade direcionada para uma câmara e metade para a outra câmara.

Trinta e duas amostras homogêneas de 40 maçãs 'Galaxy' foram selecionadas no parque de armazenagem e acondicionadas em caixas plásticas. Maçãs de 16 amostras foram tratadas com 1-MCP por 24 h (no sétimo dia após o início do carregamento das câmaras), enquanto as demais 16 amostras permaneceram não tratadas. Após $24 \mathrm{~h}$ do tratamento com 1-MCP, 16 amostras de maçãs ( 8 tratadas com 1-MCP e 8 não tratadas) foram mantidas na câmara de $\mathrm{AC}$ a $0,7^{\circ} \mathrm{C}$, e 16 amostras ( 8 tratadas com 1-MCP e 8 não tratadas) foram mantidas na câmara de $\mathrm{AC}$ a $2,0^{\circ} \mathrm{C}$.

Após 200 dias de armazenamento sob AC, seguido de dois dias em AA $\left(23 \pm 0,3{ }^{\circ} \mathrm{C} / \mathrm{UR}\right.$ de $68 \pm 6 \%$ ), os frutos foram analisados quanto a firmeza da polpa e severidade de escurecimento da polpa, podridões e rachaduras, conforme descrito no experimento 1 .

$\mathrm{O}$ consumo de energia nas câmaras a $0,7^{\circ} \mathrm{C}$ 
e 2,0 ${ }^{\circ} \mathrm{C}$ foi estimado por medidas de frequência de abertura da válvula solenoide de gás refrigerante (amônia) no evaporador, e pela medida do consumo de energia (KWH) e frequência de ventilação nos evaporadores das duas câmaras. As frequências de abertura de válvulas solenoides e de ventilação foram determinadas através de sistemas de automação industrial, com Controladores Lógicos Programáveis e softwares específicos. O consumo de energia pelos motores de ventilação foi determinado pela instalação de medidores de consumo de energia.

$\mathrm{O}$ experimento seguiu o delineamento inteiramente casualizado, com oito repetições, em esquema fatorial $(2 \times 2)$, correspondente a duas temperaturas de armazenagem $\left(0,7\right.$ e $\left.2,0^{\circ} \mathrm{C}\right)$ e duas doses de 1-MCP $\left(0\right.$ e $\left.1 \mu \mathrm{L} \mathrm{L}^{-1}\right)$.

Experimento 3: maçãs 'Gala' e 'Imperial Gala' colhidas em São Joaquim e armazenadas em câmaras comerciais

A colheita de frutos foi feita em pomares comerciais de maçãs 'Gala' (plantada em 1997, sobre porta-enxerto Marubakaido) e 'Imperial Gala' (plantada em 2004, sobre porta-enxerto EM9), localizados no município de São Joaquim, SC.

O experimento foi conduzido em três câmaras comercias de $578 \mathrm{~m}^{3}$, no parque de armazenagem de empresa localizada em São Joaquim, ajustadas nas temperaturas de $0,8 \pm 0,4{ }^{\circ} \mathrm{C}, 1,4 \pm 0,3{ }^{\circ} \mathrm{C}$ e $1,9 \pm 0,4{ }^{\circ} \mathrm{C}$, posicionadas em um corredor de 10 câmaras, todas do mesmo tamanho. As três câmaras de AC foram carregadas com 140 toneladas de maçãs 'Gala' e 'Imperial Gala', colhidas nos mesmos dias, durante quatro dias. As cargas de maçãs de cada caminhão, destinadas a essas câmaras, foram divididas, sendo $1 / 3$ direcionada para cada uma das três câmaras.

Vinte e quatro amostras homogêneas de 100 maçãs de cada cultivar ('Gala' colhida aos 142 DAPF, e 'Imperial Gala' colhida aos 139 DAPF) foram selecionadas no parque de armazenagem, pesadas e acondicionadas individualmente em caixas plásticas. Em cada cultivar, 12 amostras foram tratadas com 1-MCP por $24 \mathrm{~h}$, segundo metodologia descrita no experimento 1 , enquanto as demais 12 amostras permaneceram não tratadas. Após $24 \mathrm{~h}$ do tratamento com 1-MCP, oito amostras de maçãs de cada cultivar, quatro tratadas com 1-MCP e quatro não tratadas com 1-MCP, foram armazenadas em cada uma das três câmaras, sob condição de $\mathrm{AC}$, mantidas nas temperaturas de $0,8^{\circ} \mathrm{C}, 1,4{ }^{\circ} \mathrm{C}$ e $1,9^{\circ} \mathrm{C}$.

$\mathrm{O}$ 1-MCP foi gerado no interior das câmaras comerciais a $1,4{ }^{\circ} \mathrm{C}$ e $1,9{ }^{\circ} \mathrm{C}$, pela dissolução de ciclodextrina-1-MCP (SmartFresh ${ }^{\mathrm{TM}}$, 3,3\% i.a., AgroFresh Inc.) em água. O pó ciclodextrina1-MCP, embalado em bolsa hidrossolúvel, foi depositado em reservatório de água de um gerador de 1-MCP ${ }^{\mathrm{TM}}$ (AgroFresh Inc.). A porta da câmara de armazenagem foi lacrada 5 minutos depois da bolsa de ciclodextrina-1-MCP ter sido depositada na água, garantido que a dissolução da bolsa hidrossolúvel e a gaseificação do 1-MCP tenham ocorrido em ambiente hermético. A dissolução da ciclodextrina-1-MCP em água e a prevenção de sua precipitação foi favorecida pelo borbulhamento de ar no reservatório de água.

Após 230 dias de armazenamento, as maçãs foram mantidas sob AA $\left(23 \pm 0,3{ }^{\circ} \mathrm{C} / \mathrm{UR}\right.$ de $\left.68 \pm 6 \%\right)$, por sete dias, antes de serem analisadas quanto à firmeza da polpa e severidade de escurecimento da polpa e podridões, conforme metodologia descrita no experimento 1 .

O experimento seguiu o delineamento inteiramente casualizado, com quatro repetições, em esquema fatorial $(3 \times 2)$, correspondente a três temperaturas de armazenagem $\left(0,8 ; 1,4\right.$ e $\left.1,9^{\circ} \mathrm{C}\right)$ e duas doses de 1-MCP $\left(0\right.$ e $\left.1 \mu \mathrm{L} \mathrm{L}^{-1}\right)$.

\section{Análise estatística dos dados}

Foi realizada análise de variância (ANOVA) e teste de comparação de médias (Tukey; $p<0,05$ ), visando avaliar os efeitos dos tratamentos sobre os diferentes atributos de qualidade avaliados, utilizando o programa SAS. Dados em porcentagem foram transformados através da fórmula arco seno $[(\mathrm{x}+0,5) / 100]^{1 / 2}$, antes de serem submetidos à ANOVA.

\section{RESULTADOS E DISCUSSÃO}

Experimento 1: maçãs 'Royal Gala' colhidas em Fraiburgo e armazenadas em câmaras experimentais

Maçãs 'Royal Gala' colhidas aos 140 DAPF apresentaram maturação mais avançada (firmeza da polpa de $63,1 \mathrm{~N}$ e índice de amido de 7,9) que aquelas colhidas 124 DAPF (firmeza da polpa de 73 $\mathrm{N}$ e índice de amido de 4,4) (diferença significativa; $\mathrm{p}<0,05)$.

Maçãs colhidas aos 124 DAPF mantiveram, após a armazenagem em AC, maior firmeza da polpa que aquelas colhidas aos 140 DAPF (Tabela 1).

Não houve efeito da temperatura sobre a conservação da firmeza da polpa de maçãs colhidas aos 140 DAPF, tratadas ou não com 1-MCP. Porém, maçãs colhidas aos 124 DAPF e armazenadas em $\mathrm{AC}$ a $1,2{ }^{\circ} \mathrm{C}$ mantiveram maior firmeza da polpa que aquelas armazenadas a $-0,3{ }^{\circ} \mathrm{C}$, quando não tratadas com 1-MCP (Tabela 1). Maçãs tratadas com 1-MCP mantiveram maior firmeza da polpa quando armazenadas a $-0,3{ }^{\circ} \mathrm{C}$ nos frutos colhidos aos 124 DAPF, e a $1,2^{\circ} \mathrm{C}$ nos frutos colhidos aos 140 DAPF. 
Frutos colhidos aos 140 DAPF apresentaram de forma consistente maiores valores de percentagem de escurecimento interno em relação a frutos colhidos aos 124 DAPF (Tabela 1). Houve menor incidência de escurecimento da polpa pelo aumento da temperatura de armazenamento em AC de - 0,3 para $1,2{ }^{\circ} \mathrm{C}$, independente da data de colheita e do tratamento com 1-MCP. O tratamento com 1-MCP reduziu o desenvolvimento de escurecimento da polpa nos frutos colhidos aos 140 DAPF e armazenados a $-0,3{ }^{\circ} \mathrm{C}$.

A percentagem de maçãs com podridões após a armazenagem em $\mathrm{AC}$ reduziu com o aumento na temperatura de armazenamento de $-0,3{ }^{\circ} \mathrm{C}$ para 1,2 ${ }^{\circ} \mathrm{C}$ em frutos colhidos $124 \mathrm{DAPF}$, independentemente o tratamento com 1-MCP, e em frutos colhidos 140 DAPF, porém não tratados com 1-MCP (Tabela 1).

Apenas frutos colhidos aos 140 DAPF apresentaram rachaduras após a armazenagem em AC (Tabela 1). Nestes frutos, o tratamento com 1-MCP reduziu o desenvolvimento de rachaduras, independentemente da temperatura de armazenagem. Em frutos não tratados com 1-MCP, a incidência de rachaduras foi reduzida pelo aumento da temperatura de armazenagem.

Experimento 2: maçãs 'Galaxy' colhidas em Fraiburgo e armazenadas em câmaras comerciais

Após o período de armazenagem em $\mathrm{AC}$, maçãs 'Galaxy' armazenadas a $0,7^{\circ} \mathrm{C}$ apresentaram menor firmeza de polpa em relação às maçãs armazenadas a $2{ }^{\circ} \mathrm{C}$, quando não tratadas com 1-MCP (Tabela 2). Entretanto, quando tratados com 1-MCP, os frutos armazenados a $0,7^{\circ} \mathrm{C}$ apresentaram maior firmeza de polpa em relação aos frutos armazenados a $2{ }^{\circ} \mathrm{C}$.

A incidência de maçãs 'Galaxy' com escurecimento da polpa e rachaduras foi reduzida pelo aumento da temperatura de armazenamento em $\mathrm{AC}$ de 0,7 para $2,0^{\circ} \mathrm{C}$, para frutos não tratados com 1-MCP (Tabela 2), semelhante ao observado para frutos armazenados em câmaras experimentais. No entanto, o tratamento com 1-MCP não reduziu a incidência de escurecimento da polpa e rachaduras, independente da temperatura de armazenamento. Neste experimento, a ocorrência de rachaduras foi baixa, não sendo verificada a $2,0{ }^{\circ} \mathrm{C}$, e ocorrendo apenas em frutos armazenados a $0,7^{\circ} \mathrm{C}$ e não tratados com 1-MCP $(2,8 \%)$.

Diferente do que ocorreu em maçãs armazenadas em câmaras experimentais, a incidência de maçãs 'Galaxy' com sintomas de podridões não foi afetada pelos tratamentos de temperatura e 1-MCP, quando armazenadas em câmaras comerciais (dados não apresentados).
$\mathrm{O}$ aumento da temperatura em $\mathrm{AC}$ de 0,7 ${ }^{\circ} \mathrm{C}$ para $2,0{ }^{\circ} \mathrm{C}$ reduziu o consumo de energia em aproximadamente $21 \%$ para ventilação e $50 \%$ para refrigeração (Tabela 3). O maior consumo de energia em câmaras de armazenagem de frutas ocorre na compressão do gás refrigerante pelas máquinas de refrigeração. No entanto, a gaseificação do refrigerante (amônia) e o escoamento do calor da atmosfera de armazenagem ao ambiente externo ocorrem a partir da abertura da válvula solenoide. Assim, embora a abertura da válvula solenoide per se não represente um consumo significativo de energia elétrica, a demanda de refrigeração da atmosfera de armazenagem e a compressão do gás refrigerante estão diretamente relacionados a frequência de abertura da válvula solenoide.

Experimento 3: maçãs 'Gala' e 'Imperial Gala' colhidas em São Joaquim e armazenadas em câmaras comerciais.

A manutenção da firmeza da polpa após a armazenagem em AC foi influenciada pela temperatura apenas em maçãs 'Imperial Gala' não tratadas com 1-MCP, onde frutos armazenados a 1,9 ${ }^{\circ} \mathrm{C}$ apresentaram maior firmeza da polpa que aqueles armazenados a 1,4 ou $0,8^{\circ} \mathrm{C}$ (Tabela 4). No entanto, frutos desta cultivar armazenados a $0,8^{\circ} \mathrm{C}$ e tratados com 1-MCP apresentaram maior firmeza da polpa. Maçãs de ambas as cultivares tratadas com 1-MCP apresentaram maior firmeza da polpa que maçãs não tratadas, independentemente da temperatura de armazenagem.

Em maçãs 'Gala', a incidência de escurecimento interno foi menor em frutos armazenados em temperaturas mais altas (1,4 e 1,9 ${ }^{\circ} \mathrm{C}$ ), quando não tratados com 1-MCP (Tabela 4). Em frutos tratados com 1-MCP não houve efeito do incremento da temperatura. Contrariamente ao observado em 'Gala', na maçã 'Imperial Gala' o incremento da temperatura de armazenagem aumentou a incidência de escurecimento de polpa em frutos tratados com 1-MCP. Os resultados obtidos neste experimento com maçãs 'Gala' são semelhantes aos observados nos experimentos em câmaras comerciais com maçãs 'Galaxy' e 'Royal Gala' da região de Fraiburgo. O 1-MCP reduziu o escurecimento da polpa nas maçãs 'Gala', independentemente da temperatura de armazenagem, e em maçãs 'Imperial Gala' armazenadas a 0,8 e 1,4 ${ }^{\circ} \mathrm{C}$ (Tabela 4).

Não houve efeito da temperatura de armazenagem e do tratamento com 1-MCP na incidência de maçãs 'Gala' e 'Imperial Gala' com podridões (dados não apresentados). 
Os resultados desse estudo mostram que a temperatura de armazenagem afeta a conservação da qualidade de maçãs 'Gala' e seus clones durante a armazenagem refrigerada sob $\mathrm{AC}$, porém seus efeitos dependem do tratamento com 1-MCP e da origem dos frutos.

A manutenção da firmeza de polpa após armazenagem em AC, em geral, foi maior nos frutos armazenados em temperaturas mais altas, embora esse efeito tenha sido menos pronunciado quando os frutos foram tratados com 1-MCP. Isto contraria, em parte, os resultados obtidos em outro estudo, que apontou a temperatura de $0,5{ }^{\circ} \mathrm{C}$ como adequada para a melhor conservação da firmeza de polpa em maçãs 'Gala', em relação a temperatura de $2,5^{\circ} \mathrm{C}$, em condição de AC (BRACKMANN et al., 1996). O 1-MCP teve efeito positivo sobre a conservação da firmeza de polpa em maçãs, concordando com os resultados reportados por diversos autores (McCORMICK et al., 2010 e 2012; WATKINS, 2006 e 2008; WATKINS; MILLER, 2005). O aumento da conservação da firmeza de polpa nas maçãs tratadas com 1-MCP está diretamente relacionado à redução na ação do etileno (WATKINS, 2008).

Embora maçãs sejam consideradas tolerantes ao frio em relação a frutos de outras espécies (WANG, 2004), vários distúrbios fisiológicos que ocorrem durante a armazenagem de maçãs têm sido descritos como danos por frio. Danos por frio em maçãs podem se manifestar como escurecimento do córtex ("internal browning"), coração amarronzado ("brown core" ou "core flush"), polpa farinácea e/ ou escurecida ("low-temperature breakdown") e escurecimento da epiderme ("soft scald") (WANG, 2004).

De forma geral, em todos os experimentos, o aumento da temperatura de armazenamento em $\mathrm{AC}$, em frutos não tratados com 1-MCP, resultou em menor incidência de frutos com escurecimento da polpa. Esse resultado é semelhante aos reportados por outros autores em maçãs 'Gala' (BRACKMANN et al.,1996) e 'Empire' (JAMES et al., 2010; WATKINS, 2010), mostrando redução no escurecimento de polpa com o aumento na temperatura de armazenamento em $\mathrm{AC}$ de $0-0,5{ }^{\circ} \mathrm{C}$ para $1,7-2,2{ }^{\circ} \mathrm{C}$. Altos níveis de $\mathrm{CO}_{2}$ ocasionam o desenvolvimento de escurecimento de polpa em maçãs armazenadas em $\mathrm{AC}$, porém o aumento na temperatura de armazenamento tem um efeito determinante na redução do distúrbio (WATKINS, 2010). A ocorrência de escurecimento de polpa em maçãs é reduzida com o retardo no estabelecimento da condição de AC (JAMES et al., 2010) e com o aquecimento intermitente dos frutos (WATKINS, 2010), mostrando que o distúrbio é uma manifestação de injúria por frio. Porém, as bases fisiológicas do desenvolvimento deste distúrbio em maçãs ainda são pouco conhecidas (JAMES et al., 2010).

Frutos colhidos aos 140 DAPF (em estádio mais avançado de maturação) apresentaram maior incidência de escurecimento interno que os frutos colhidos 124 DAPF, principalmente quando armazenados a $1,2{ }^{\circ} \mathrm{C}$ e não tratados com 1-MCP. De acordo com Meheriuk et al. (1994), o escurecimento interno ("core flush") pode também estar relacionado com a senescência dos frutos, que ocorre em frutos colhidos em estádio mais avançado de maturação, submetidos ao armazenamento prolongado, em temperaturas mais elevadas $\left(1,2{ }^{\circ} \mathrm{C}\right)$ e sem o tratamento com 1-MCP, e, neste caso, não devido a uma injúria por frio.

O tratamento com 1-MCP teve efeito positivo na redução da incidência de escurecimento interno em maçãs (AMARANTE et al., 2010; WATKINS, 2006). O fato do 1-MCP reduzir o escurecimento da polpa de maçãs na maioria dos experimentos do presente estudo, indica que o desenvolvimento desse distúrbio é, em parte, uma expressão de senescência. Portanto, os resultados dos diferentes experimentos mostram que o armazenamento refrigerado de maçãs 'Gala' em AC pode ocasionar o desenvolvimento de distúrbios fisiológicos relacionados à senescência, em temperaturas de 1,2 a $2,0{ }^{\circ} \mathrm{C}$, ou induzir o desenvolvimento de distúrbios fisiológicos associados ao dano por frio, em temperaturas de $-0,3$ a $0,8{ }^{\circ} \mathrm{C}$. Assim, os resultados do presente estudo indicam que o escurecimento da polpa de maçãs 'Gala' possivelmente se deve a uma associação de senescência e dano por frio.

Os efeitos da temperatura de armazenamento e do tratamento com 1-MCP sobre a incidência de podridões não foram consistentes nos diferentes experimentos. Tem sido demonstrado que o armazenamento na temperatura de $2,5{ }^{\circ} \mathrm{C}$, durante os 40 dias iniciais, reduz a incidência de podridões, após longos períodos de armazenamento a $0{ }^{\circ} \mathrm{C}$ (BRACKMANN et al., 2000), e que o armazenamento em temperaturas abaixo de 0 ${ }^{\circ} \mathrm{C}\left(-0,8^{\circ} \mathrm{C}\right)$ agrava a incidência de podridões (BRACKMANN et al., 2010). O 1-MCP pode reduzir a incidência de podridões pós-colheita em maçãs ‘Golden Delicious' (WATKINS, 2006 e 2008). Porém, um estudo recente afirma que o 1-MCP tem efeito nulo sobre a incidência de podridões durante o armazenamento de maçãs, sugerindo que diferenças na temperatura de armazenamento pode ser o principal fator que influencia na incidência de podridões (ERRAMPALLI et al., 2012). 
A incidência de rachaduras foi mais evidente em frutos armazenados em temperaturas mais baixas e colhidos tardiamente. O tratamento com 1-MCP efetivamente reduziu a incidência de rachaduras após armazenagem em AC. Outros autores reportaram o aumento da incidência de rachaduras em frutos colhidos tardiamente (MEHERIUK et al., 1994) e a eficácia do tratamento com 1-MCP na redução deste distúrbio (WATKINS; MILLER, 2005).

$\mathrm{O}$ aumento da temperatura de $0,7^{\circ} \mathrm{C}$ para $2,0{ }^{\circ} \mathrm{C}$ resultou na redução de consumo de energia em aproximadamente $21 \%$ para ventilação e $50 \%$ para refrigeração. McCormick et al. (2012) também constataram que o aumento temperatura em $2,5^{\circ} \mathrm{C}$ (de $1,5^{\circ} \mathrm{C}$ para $4,0^{\circ} \mathrm{C}$ ) em maçãs 'Gala' resultou na redução de $35 \%$ no consumo de energia.

Um dos problemas que mais afetam a qualidade de conservação pós-colheita de maçãs 'Gala' e seus clones é a incidência de escurecimento interno. Os resultados obtidos neste estudo demostram os potenciais benefícios do aumento da temperatura de armazenagem em AC para a redução da incidência de danos por frio e conservação da qualidade de maçãs 'Gala', além de reduzir substancialmente os gastos de energia para refrigeração.

TABELA 1 - Firmeza de polpa, incidência (\%) de escurecimento interno, podridões e rachaduras em maçãs 'Royal Gala', após 210 dias de armazenagem sob atmosfera controlada, a temperaturas de $-0,3$ e $1,2^{\circ} \mathrm{C}$, mais sete (em frutos colhidos aos 124 dias após a plena floração; $124 \mathrm{DAPF}$ ) ou três (em frutos colhidos aos 140 DAPF) dias sob atmosfera do ar a $23{ }^{\circ} \mathrm{C}$. Frutos colhidos em Fraiburgo-SC, não tratados ou tratados com 1-metilciclopropeno (1-MCP), e armazenados em câmara experimental de $0,150 \mathrm{~m}^{3}$.

\begin{tabular}{|c|c|c|c|c|c|c|c|c|}
\hline \multirow{2}{*}{$\begin{array}{c}\text { Temperatura } \\
\left({ }^{\circ} \mathrm{C}\right)\end{array}$} & \multicolumn{2}{|c|}{124 DAPF } & $\mathbf{M}^{/ 2}$ & \multicolumn{2}{|c|}{$140 \mathrm{DAPF}$} & \multirow[t]{2}{*}{$\mathbf{M}^{/ 2}$} & \multicolumn{2}{|c|}{$\mathbf{C}^{1 / 3}$} \\
\hline & Sem 1-MCP & Com 1-MCP & $-\mathbf{M}^{2}$ & \multicolumn{2}{|c|}{ Sem 1-MCP Com 1-MC } & & \multicolumn{2}{|c|}{ Sem 1-MCP Com 1-MCP } \\
\hline & \multicolumn{8}{|c|}{ Firmeza de polpa $(\mathrm{N})$} \\
\hline$-0,3$ & 52,0 & 58,2 & $* *$ & 42,7 & 45,8 & ns & $* * *$ & $* * *$ \\
\hline \multirow[t]{3}{*}{1,2} & 58,2 & 61,3 & ns & 43,1 & 47,1 & $* *$ & $* * *$ & $* * *$ \\
\hline & ** & ns & & ns & ns & & & \\
\hline & \multicolumn{8}{|c|}{ Escurecimento interno (\%) } \\
\hline$-0,3$ & 38,1 & 42,4 & ns & 66,5 & 55,5 & $* * *$ & $* * *$ & ns \\
\hline \multirow[t]{3}{*}{1,2} & 25,7 & 23,2 & ns & 42,5 & 34,2 & ns & $* *$ & $* * *$ \\
\hline & $* *$ & $* * *$ & & $* * *$ & $*$ & & & \\
\hline & \multicolumn{8}{|c|}{ Podridões $(\%)$} \\
\hline$-0,3$ & 31,9 & 22,1 & * & 20,5 & 13,0 & $*$ & $* * *$ & $* *$ \\
\hline \multirow[t]{3}{*}{1,2} & 4,9 & 3,2 & ns & 7,5 & 12,0 & ns & ns & $* *$ \\
\hline & $* * *$ & $* * *$ & & $* * *$ & ns & & & \\
\hline & \multicolumn{8}{|c|}{ Rachaduras (\%) } \\
\hline$-0,3$ & 0,0 & 0,0 & ns & 22,0 & 3,0 & $* * *$ & $* * *$ & $*$ \\
\hline 1,2 & 0,0 & 0,0 & ns & 10,0 & 2,0 & $* * *$ & $* * *$ & ns \\
\hline & ns & ns & & $* * *$ & ns & & & \\
\hline
\end{tabular}

${ }^{1 /}$ Efeito da temperatura $(\mathrm{T})$.

${ }^{12}$ Efeito do tratamento com 1-MCP (M).

${ }^{13}$ Efeito da data de colheira (C).

$*, * * \mathrm{e}^{* * *}=$ significativo a $5 \%, 1 \%$ e $0,1 \%$, respectivamente. $\mathrm{ns}=$ não significativo. 
TABELA 2 - Firmeza de polpa e incidência (\%) de escurecimento da polpa e de rachaduras em maçãs 'Galaxy' colhidas em Fraiburgo-SC, não tratados ou tratados com 1-metilciclopropeno (1MCP), e armazenadas sob atmosfera controlada, em câmara comercial de $2050 \mathrm{~m}^{3}$, durante 200 dias, nas temperaturas de 0,7 e $2,0^{\circ} \mathrm{C}$, seguido de dois dias sob atmosfera do ar a $23^{\circ} \mathrm{C}$.

\begin{tabular}{|c|c|c|c|c|c|c|c|c|c|}
\hline \multicolumn{3}{|c|}{ Temperatura Sem 1-MCP Com 1-MCP } & \multirow{2}{*}{\multicolumn{4}{|c|}{$\mathbf{M}^{/ 2} \frac{\text { Sem 1-MCP Com 1-MCP }}{\text { Escurecimento interno (\%) }} \mathbf{M}$}} & \multicolumn{3}{|c|}{ Sem 1-MCP Com 1-MCP } \\
\hline$\left({ }^{\circ} \mathrm{C}\right)$ & Firmez & $\operatorname{lpa}(\mathrm{N})$ & & & & & $\mathrm{Rac}$ & $(\%)$ & \\
\hline 0,7 & 38,2 & 46,7 & $* * *$ & 39,4 & 33,5 & ns & 2,8 & 0,0 & ns \\
\hline 2,0 & 44,0 & 43,5 & ns & 21,5 & 24,7 & ns & 0,0 & 0,0 & ns \\
\hline & $* * *$ & $*$ & & $* *$ & ns & & $*$ & ns & \\
\hline
\end{tabular}

${ }^{1}$ Efeito da temperatura (T).

${ }^{2}$ Efeito do tratamento com 1-MCP (M)

$*, * * \mathrm{e}^{* * *}=$ significativo a $5 \%, 1 \%$ e $0,1 \%$, respectivamente. $\mathrm{ns}=$ não significativo.

TABELA 3 - Frequência de acionamento do sistema de refrigeração, consumo de energia para ventilação da câmara e economia de energia para refrigeração e ventilação em câmaras comerciais de 2050 $\mathrm{m}^{3}$, em Fraiburgo-SC, após 195 dias de armazenagem sob atmosfera controlada.

\begin{tabular}{|c|c|c|c|c|}
\hline \multirow{2}{*}{ Mês(es) } & \multirow{2}{*}{$\begin{array}{r}\text { Número } \\
\text { de dias }\end{array}$} & \multicolumn{2}{|c|}{ Temperatura } & \multirow{2}{*}{$\begin{array}{l}\text { Economia de } \\
\text { Energia }(\%)\end{array}$} \\
\hline & & $0,7^{\circ} \mathrm{C}$ & $2,0^{\circ} \mathrm{C}$ & \\
\hline & & \multicolumn{2}{|c|}{$\begin{array}{c}\text { Resfriamento } \\
\text { (frequência de abertura da válvula solenoide) }\end{array}$} & \\
\hline Fev. e Mar. & 35 & 6159 & 3796 & 38,4 \\
\hline Abr. & 30 & 5128 & 3462 & 32,5 \\
\hline Maio & 31 & 7097 & 3127 & 55,9 \\
\hline Jun. & 30 & 6386 & 2384 & 62,7 \\
\hline Jul. & 31 & 5277 & 2416 & 54,2 \\
\hline Ago. e Set. & 38 & 6453 & 3492 & 45,9 \\
\hline Total & & 36500 & 18677 & 48,8 \\
\hline
\end{tabular}

Ventilação (KWH)

\begin{tabular}{llllc} 
Fev. e Mar. & 35 & 3364 & 1894 & 43,7 \\
Abr. & 30 & 1568 & 1289 & 17,8 \\
Maio & 31 & 1481 & 1185 & 20,0 \\
Jun. & 30 & 1325 & 1204 & 9,1 \\
Jul. & 31 & 1494 & 1388 & 7,1 \\
Ago. e Set. & 38 & 2085 & 1947 & 6,6 \\
Total & & $\mathbf{1 1 3 1 7}$ & $\mathbf{8 9 0 7}$ & $\mathbf{2 1 , 3}$ \\
\hline
\end{tabular}


TABELA 4 - Firmeza de polpa e incidência (\%) de escurecimento interno em maçãs 'Gala' ' 'Imperial Gala', após 230 dias de armazenagem sob atmosfera controlada, a temperaturas de $0,8,1,4$ e $1,9^{\circ} \mathrm{C}$, mais sete dias sob atmosfera do ar a $23^{\circ} \mathrm{C}$. Frutos colhidos em São Joaquim-SC, não tratados ou tratados com 1-metilciclopropeno (1-MCP), e armazenados em câmara comercial de $578 \mathrm{~m}^{3}$.

\begin{tabular}{|c|c|c|c|c|c|c|}
\hline \multirow{2}{*}{$\begin{array}{c}\text { Temperatura } \\
\left({ }^{\circ} \mathrm{C}\right)\end{array}$} & Sem 1-MCP & Com 1-MCP & \multirow[t]{2}{*}{$\mathrm{M}^{/ 1}$} & Sem 1-MCP & Com 1-MCP & \multirow[t]{2}{*}{$\mathrm{M}^{/ 1}$} \\
\hline & \multicolumn{2}{|c|}{ Firmeza de polpa $(\mathrm{N})$} & & \multicolumn{2}{|c|}{ Escurecimento interno (\%) } & \\
\hline & \multicolumn{6}{|c|}{ 'Gala' } \\
\hline 0,8 & 49,3 a & $71,1 \mathrm{a}$ & $* * *$ & $45,3 \mathrm{a}$ & $5,3 \mathrm{a}$ & $* * *$ \\
\hline 1,4 & 49,8 aа & $69,3 \mathrm{a}$ & $* * *$ & $34,3 \mathrm{~b}$ & $4,1 \mathrm{a}$ & $* * *$ \\
\hline \multirow[t]{2}{*}{1,9} & 50,7 aa & 68,9 a & $* * *$ & $37,8 \mathrm{~b}$ & $7,8 \mathrm{a}$ & $* * *$ \\
\hline & \multicolumn{6}{|c|}{ 'Imperial Gala' } \\
\hline 0,8 & $51,1 \mathrm{~b}$ & $71,5 \mathrm{a}$ & $* * *$ & 28,9 a & $15,5 \mathrm{~b}$ & $* *$ \\
\hline 1,4 & $52,9 \mathrm{ab}$ & $67,5 \mathrm{~b}$ & $* * *$ & 25,3 a & $17,8 \mathrm{ab}$ & $*$ \\
\hline 1,9 & $55,5 \mathrm{a}$ & $69,3 \mathrm{~b}$ & $* * *$ & $23,3 \mathrm{a}$ & $21,0 \mathrm{a}$ & ns \\
\hline
\end{tabular}

${ }^{1}$ Efeito do tratamento com 1-MCP (M).

$*, * * \mathrm{e}^{* * *}=$ significativo a $5 \%, 1 \%$ e $0,1 \%$, respectivamente. ns = não significativo.

Médias seguidas de mesma letra, nas colunas, em uma mesma cultivar, diferem entre si pelo teste de Tukey $(\mathrm{p}<0,05)$.

\section{CONCLUSÕES}

Em maçãs clones de 'Gala' armazenadas em condição de atmosfera controlada:

A utilização de temperaturas de armazenagem de 1,2 a $2,0{ }^{\circ} \mathrm{C}$ reduz de forma consistente a perda da firmeza da polpa e a ocorrência de escurecimento de polpa, rachaduras e podridões, em relação a temperaturas de $-0,3$ a $0,8{ }^{\circ} \mathrm{C}$, especialmente em frutos não tratados com 1-MCP.

$\mathrm{O}$ tratamento com 1-MCP reduz de forma consistente a perda de firmeza de polpa e a ocorrência de escurecimento de polpa e rachaduras;

O aumento da temperatura de armazenamento de 0,7 para $2,0^{\circ} \mathrm{C}$, reduz o consumo de energia em aproximadamente $21 \%$ para ventilação e $50 \%$ para refrigeração.

\section{REFERÊNCIAS}

AMARANTE, C.V.T.; ARGENTA, L.C.; VIEIRA, M.J.; STEFFENS, C.A. Alteração da eficiência do 1-MCP com o retardo na sua aplicação após a colheita em maçãs 'Fuji Suprema'. Revista Brasileira de Fruticultura, Jaboticabal, v.32, n.4, p.984-992, 2010.
BRACKMANN, A.; ARGENTA, L.C.; MAZARO, S.M. Concentrações de $\mathrm{O}_{2}$ e $\mathrm{CO}_{2}$ na qualidade de maçãs (Malus domestica Bork) cv. Gala, armazenadas a 0,5 e $2,5^{\circ} \mathrm{C}$. Revista Brasileira de Agrociência, Pelotas, v.2, n.1, p.51-56, 1996.

BRACKMANN, A.; BOTH, V.; WEBER, A.; PAVANELLO, E.P.; SCHORR, M.R.W.; SANTOS, J.R.A. Variação da temperatura, oxigênio e $\mathrm{CO}_{2}$ durante o armazenamento em atmosfera controlada de maçãs 'Royal Gala'. Semina: Ciências Agrárias, Londrina, v.33, n.6, p.2247-2256, 2012.

BRACKMANN, A.; CERETTA, M.; PINTO, J.A.V.; VENTURINI, T.L.; LÚCIO, A.D.C. Tolerância de maçã 'Gala' a baixas temperaturas durante o armazenamento. Ciência Rural, Santa Maria, v.40, n.9, p.1909-1915, 2010.

BRACKMANN, A.; HUNSCHE, M.; STEFFENS, C.A. Conservação da maçã 'Fuji' sob diferentes temperaturas, umidades relativas e momentos de instalação da atmosfera de armazenamento. Ciência Rural, Santa Maria, v.30, n.1, p.81-84, 2000.

BROWN, G. Saving apple storage costs. Australian Fruit Grower, Melbourne, v.5, n.1, p.19-21, 2011. 
EAST, A.R.; SMALE, N.J.; TRUJILLO, F.J. Potential for energy cost savings by utilizing alternative temperature control strategies for controlled atmosphere stored apples. International Journal of Refrigeration, Berlin, v.36, n.3, p.11091117, 2013.

ERRAMPALLI, D.; WAINMAN, L.I.; DeELL, J.R. Reduced risk control options for apple postharvest diseases in long-term storages. Acta Horticulturae, Leuven, n.934, p.313-318, 2012.

JAMES, H.J.; NOCK, J.F.; WATKINS, C.B. The failure of postharvest treatments to control firm flesh browning in Empire apples. New York Fruit Quarterly, Nova York, v.18, n.3, p.5-8, 2010.

KWEON, H-J.; KANG, I-K.; KIM, M-J.; LEE, J.; MOON, Y-S.; CHOI, C.; CHOI, D.G.; WATKINS, C.B. Fruit maturity, controlled atmosphere delays and storage temperature affect fruit quality and incidence of storage disorders of 'Fuji' apples. Scientia Horticulturae, Amsterdam, v.157, n.1, p.60-64, 2013.

LIU, Y.; LANGER, V.; HOGH-JENSEN, H.; EGELYNG, H. Life cycle assessment of fossil energy use and greenhouse gas emissions in Chinese pear production. Journal of Cleaner Production, Knoxville, v.18, n.14, p.1423-1440, 2010.

McCORMICK, R.; NEUWALD, D.A.; STREIF, J. A case study: Potential energy savings using 1-MCP with 'Gala' apples in commercial CA storage. Acta Horticulturae, Leuven, n.877, p.323-326, 2010. (1)

McCORMICK, R.; NEUWALD, D.A.; STREIF, J. Commercial apple $\mathrm{CA}$ storage temperature regimes with 1-MCP (Smart Fresh ${ }^{\mathrm{TM}}$ ): Benefits and risks. Acta Horticulturae, Leuven, n.934, p.263-270, 2012.

MEHERIUK, M., PRANGE, R.K.; LIDSTER, P.D.; PORRITT, S.W. Postharvest disorders of apples and pears. Ottawa: Agriculture Canada, 1994. 66p. (Publication, 1737E).

PRANGE, R.K.; DeLONG, J.M.; DANIELS-LAKE, B.J.; HARRISON, P.A. Innovation in controlled atmosphere technology. Stewart Postharvest Review, London, v.1, n.3, p.1-14, 2005.
WANG, C.Y. Chilling and freezing injury. The commercial storage of fruits, vegetables, and florist and nursery stocks. Washington: United States Department of Agriculture, 2004. (Agriculture Handbook, 66)

WATKINS, C.B. Overview of 1-methylcyclopropene trials and uses for edible horticultural crops. HortScience, Alexandria, v.43, n.1, p.86-94, 2008.

WATKINS, C.B. Storage disorders of controlled atmosphere-stored Empire apples. New York Fruit Quarterly, Nova York, v.18, n.3, p.19-22, 2010.

WATKINS, C.B. The effect of 1-MCP on the development of physiological storage disorders in horticultural crops. Stewart Postharvest Review, London, v.3, n.2, p.1-6, 2007.

WATKINS, C.B. The use of 1-methylcyclopropene (1-MCP) on fruits and vegetables. Biotechnology Advances, Kidlington, v.24, n.1, p.389-409, 2006.

WATKINS, C.B.; KUPFERMAN, E.; ROSENBERGER, D.A. Apple. The commercial storage of fruits, vegetables, and florist and nursery stocks. Washington: United States Department of Agriculture, 2004. (Agriculture Handbook, 66))

WATKINS, C.B.; MILLER, W.B. A summary of physiological processes or disorders in fruits, vegetables and ornamental products that are delayed or decreased, increased, or unaffected by application of 1-metylcyclopropene (1MCP). New York: Department of Horticulture, Cornell University, Ithaca, 2005. Disponível em: $<$ http://www.hort.cornell.edu/mcp/ $>$. Acesso em: 18 jul. 2013. 